\title{
A Review on Recent Trends in the Development of Gastro Retentive Floating Drug Delivery System
}

\author{
Seema Mahor ${ }^{1 *}$, Neel Kant Prasad ${ }^{2}$, Phool Chandra ${ }^{3}$ \\ ${ }^{\text {I}}$ Department of Pharmaceutical Sciences, Vishveshwarya Group of Institutions, Greater Noida, 203207, U.P. India \\ ${ }^{2}$ S.G.T. College of Pharmacy, SGT University, Gurgaon \\ ${ }^{3}$ IFTM University Moradabad, U.P. India
}

Address for Correspondence: Seema Mahor, seemamahor@gmail.com

\section{Received: \\ 01.06.2018 \\ Accepted:}

25.08.2018

Keywords Gastric Retention Time; Floating Drug Delivery System; in-vitro evaluation.

\begin{abstract}
Floating microspheres are also known as hollow microspheres; they are gastro-retentive drug delivery systems which are based on non-effervescent approach. Floating microspheres are in strict sense, spherical empty particles without core, free flowing powders consisting of proteins or synthetic polymers, ideally having a size in the range 1-1000 micro meter. Gastro-retentive floating microspheres are low-density systems that have sufficient buoyancy to float over gastric contents and remain in stomach for prolonged period. The drug is released slowly at desired rate resulting in increased gastric retention with reduced fluctuations in plasma drug concentration. Floating microspheres having various advantages over conventional dosage forms like, they can be used to improve patient compliance by decreasing dosing frequency, better therapeutic effect of short half-life drugs can be achieved, Enhanced absorption of drugs which solubilises only in stomach, Gastric retention time is increased because of buoyancy. Floating microspheres are prepared by solvent diffusion and evaporation methods to create the hollow inner core. In the present review preparation, methods, characterization, advantages, mechanism of drug release from microspheres, list of polymers, applications and list of the drugs formulated as floating microspheres are discussed. () 2019 iGlobal Research and Publishing Foundation. All rights reserved.
\end{abstract}

Cite this article as: Mahor, S.; Prasad, N.K.; Chandra, P. A review on recent trends in the development of gastro retentive floating drug delivery system. Indo Global J. Pharm. Sci., 2019; 9(1): 13-24. DOI: http://doi.org/10.35652/IGJPS.2019.9103.

\section{INTRODUCTION}

The goal of any drug delivery system is to provide a therapeutic amount of drug to the proper site in the body to achieve promptly and then maintain the desired drug concentration. The most convenient and commonly employed route of drug delivery has historically been by oral ingestion. Oral delivery of the drug is by far the most preferable route of drug delivery due to various reasons like- ease of administration, patient compliance and flexibility in the formulation. From immediate release to site-specific delivery, oral dosage form has really progressed. It is evident from the recent scientific and patented literature that an increased interest in novel dosage forms that are retained in the stomach for the prolonged and predicted period of time exists today in academic and industrial research groups [1].

Development of oral controlled-release systems now a day has been a challenge to formulation scientists because of their inability to retain and localize the dosage form in the targeted area of the gastrointestinal tract. These controlled/sustained release preparations using alternative routes have been formulated but the oral route still remains preferable [2]. 
Indo Global Journal of Pharmaceutical Sciences, 2019; 9(1): 13-24

Single-unit formulations are associated with some problem being obstructed in the gastrointestinal tract, which may have a potential danger of producing gastric irritation. But a floating drug delivery system made of multiple-unit forms has various advantages over the single unit dosage forms. On each subsequent gastric empting, particles will spread out over a large area of absorption sites, increasing the opportunity for drug release profile and absorption in a more or less predictable way [3]. Oral controlled release dosage form shows the drug absorption at desired rate means, to reach effective plasma levels within an acceptable short time period, next to avoid an overshoot in the case of rapidly absorbed drugs and to maintain affective plasma levels over the desired time period [4]. Recent advances indicate that floating microspheres are especially suitable for achieving sustained release oral dosage form with flexibility of blending to attain different release patterns, low risk of dose dumping as well as reproducible and increase in the gastric residence time[5]. Hollow microspheres are spherical empting particles without core. These microspheres are characteristically free flowing powder in nature consisting of proteins or synthetic polymers. Gastro-retentive floating microspheres are low density systems that have sufficient buoyancy to float over a gastric content and remain buoyant for prolonged period of time [6]. Gastric emptying of dosage form is extensively variable process and ability to prolong and control the emptying time is valuable asset for dosage forms, which reside in the stomach for a prolong period of time than a conventional dosage forms. Several difficulties are faced in designing controlled released system for better absorption and enhanced bioavailability. Conventional oral dosage forms like tablets, capsules provides specific drug concentration in systemic circulation without offering any control over drug delivery and also cause great fluctuations in plasma drug levels. Single unit dosage forms have disadvantage of a release all or nothing during empting process while the multi-unit particulate system pass through the GIT to avoid the vagaries of gastric emptying and thus release the drug more uniformly. The uniform distribution of these multi-unit dosage forms along the GIT could result in more reproducible drug absorption and reduced risk of local irritations. This floating dosage forms enhances bioavailability being locally active in the stomach and or upper part of the intestine. [7, 8]

\section{APPROACHES TO GASTRIC RETENTION [8,9]}

A number of approaches have been used to increase gastric retention time (GRT) of a dosage form in stomach by employing a variety of concepts.

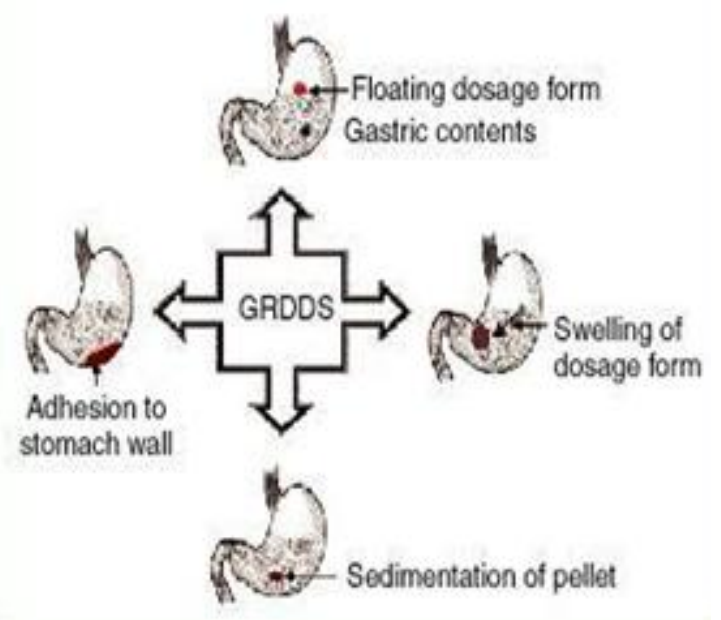

Figure 1. Types of Gastro-retentive drug delivery systems [8]

Floating systems: Floating Drug Delivery Systems (FDDS) have a bulk density lower than gastric fluids and thus remain buoyant in stomach for a prolonged period of time, without affecting the gastric emptying rate. While the system floats on gastric contents, the drug is released slowly at a desired rate from the system. After the release of drug, the residual system is emptied from the stomach. This results in an increase in gastric retention time and a better control of fluctuations in plasma drug concentrations. Floating drug systems can be classified into two distinct categories, non-effervescent and effervescent systems.

Bio/muco-adhesive systems: Bio/muco-adhesive systems are those system which bind to the gastric epithelial cell surface or mucin and enhance the gastric residence time of the dosage form in stomach, by increasing the intimacy and duration of contact of drug with the biological membrane. Binding of polymers to mucin or epithelial surface can be divided into three broad categories:

- Hydration-mediated adhesion.

- Bonding-mediated adhesion.

- Receptor-mediated adhesion.

Swelling and expanding systems: These are dosage forms, which after swallowing swell in the gastric fluid to an extent that prevents their exit from the pylorus. As a result, the dosage form is retained in stomach for a long period of time. These systems may be named as "plug type system", since they exhibit tendency to remain logged at the pyloric sphincter.

High density systems: These systems having high density of about $3 \mathrm{~g} / \mathrm{cm} 3$ are retained in the stomach and are capable of 


\section{Indo Global Journal of Pharmaceutical Sciences, 2019; 9(1): 13-24}

withstanding its peristaltic movements. A density of 2.6- 2.8 $\mathrm{g} / \mathrm{cm} 3$ acts as a threshold value after which such systems can be retained in the lower parts of the stomach. High-density systems include coated pellets. Coating is done by heavy inert material such as barium sulphate, zinc oxide, titanium dioxide, iron powder etc.

Incorporation of passage delaying food agents; Food excipients like fatty acids e.g. salts of myristic acid change and modify the pattern of stomach to a fed state, thereby decreasing gastric emptying rate and permitting considerable prolongation of release. The delay in gastric emptying after meals rich in fats is largely caused by saturated fatty acids with chain length of $\mathrm{C} 10-\mathrm{C} 14$.

Ion exchange resins: Ion exchange resins are loaded with bicarbonate and a negatively charged drug is bound to the resin. The resultant beads are then encapsulated in a semipermeable membrane to overcome the rapid loss of carbon dioxide. Upon arrival in the acidic environment of the stomach, an exchange of chloride and bicarbonate ions take place. As a result of this reaction carbon dioxide is released and trapped in the membrane thereby carrying beads towards the top of gastric content and producing a floating layer of resin beads in contrast to the uncoated beads, which will sink quickly.

Osmotic regulated systems: It is an osmotic pressure controlled drug delivery device and an inflatable floating support in a bioerodible capsule. In the stomach the capsule quickly disintegrates to release the intragastric osmotic ally controlled drug delivery device. The inflatable support inside forms a deformable hollow polymeric bag that contains a liquid that gasifies at body temperature to inflate the bag. The osmotic controlled drug delivery device consists of two components - drug reservoir compartment and osmotically active compartment.

\section{FACTORS AFFECTING GASTRIC RETENTION [10]}

The stomach anatomy and physiology contain parameters to be considered in the development of gastro retentive dosage forms. To pass through the pyloric valve in to the small intestine the particle size should be in the range of 1 to $2 \mathrm{~mm}$. The most important parameters controlling the gastric retention time (GRT) of oral dosage forms include: density, size and shape of the dosage form, food intake and its nature, caloric content and frequency of intake, posture, gender, age, sex, sleep, body mass index, physical activity and diseased states of the individual ( e.g. chronic disease, diabetes etc.) and administration of drugs with impact on gastrointestinal transit time for example drugs acting as anti cholinergic agents ( e.g. atropine, propantheline), Opiates ( e.g. codeine) and prokinetic agents ( e.g. metclopramide, cisapride.). The molecular weight and lipophilicity of the drug depending on its ionization state are also important parameters.

The density of a dosage form: GRT is a function of dosage form buoyancy that is dependent on the density. [38]. Dosage forms having a density lower than the gastric contents can float to the surface of gastric content, while high density systems sink to bottom of the stomach. Both positions may isolate the dosage system from the pylorus. A density of $<1.0$ $\mathrm{gm} / \mathrm{cm} 3$ is required to exhibit floating property [10].

Shape and size of the dosage form: Dosage form which have a diameter of more than $7.5 \mathrm{~mm}$ are reported to have an increased GRT as compared to those with a diameter of $9.9 \mathrm{~mm}$ [38]. Shape and size of the dosage forms are important in designing indigestible single unit solid dosage forms. The mean gastric residence times of non-floating dosage forms are highly variable and greatly dependent on their size, which may be large, medium and small units. In most cases, the larger the dosage form the greater will be the gastric retention time (GRT) due to the larger size of the dosage form would not allow this to quickly pass through the pyloric ant rum into the intestine. . Ring-shaped and tetrahedron-shaped devices have a better gastric residence time as compared with other shapes.

Food intake and its nature: Food intake, viscosity and volume of food, caloric value and frequency of feeding have a profound effect on the gastric retention of dosage forms. The presence or absence of food in the gastrointestinal tract (GIT) influences the gastric retention time (GRT) of the dosage form. Usually the presence of food in the gastrointestinal tract (GIT) improves the gastric retention time (GRT) of the dosage form and thus, the drugs absorption increases by allowing its stay at the absorption site for a longer period. Again, increase in acidity and caloric value shows down gastric emptying time (GET), which can improve the gastric retention of dosage forms.

\section{ADVANTAGES OF GASTRO RETENTIVE DOSAGE FORM $[11,37]$}

Floating microspheres offers several advantages like:

- Improves patient compliance by reducing dosing frequency.

- Bioavailability enhances despite first pass effect because fluctuations in plasma drug concentration is 
Indo Global Journal of Pharmaceutical Sciences, 2019; 9(1): 13-24

avoided, a desirable plasma drug concentration is maintained by continuous drug release.

- Batter therapeutic effect of short half life drugs can be achieved.

- Gastric retention time is increased because of buoyancy.

- Drug releases in a controlled manner for a prolonged period.

- Site specific drug delivery to the stomach can be achieved i.e. antacids.

- Enhanced absorption of drugs which solubilise only in the stomach.

- Superior to single unit floating dosage forms such as microspheres releases drug uniformly and no risk of dose dumping.

- Avoidance of gastric irritation, because of sustained release effect, floatability and uniform release of drug through multi-particulate system.

- The gastro-retentive systems are advantageous for drugs absorbed through the stomach, e.g. ferrous salts, antacids. Acidic substances like aspirin cause irritation on the stomach wall when come in contact with it. Hence, HBS formulation may be useful for the administration of aspirin and other similar drugs.

\section{DISADVANTAGES OF GASTRO RETENTIVE DOSAGE FORM [12]}

- Unsuitable for drugs with limited acid solubility. E.g. Phenytoin.

- Unsuitable for drugs those are unstable in acidic environment. E.g. Erythromycin.

- Drugs that irritates or causes gastric lesions on slow release. E.g. Aspirin \&NSAIDs.

- Drugs that absorb selectively in colon E.g. Corticosteroids

\section{Drugs that can be formulated into gastro retentive dosage} form include $[10,13]$

- Drugs acting locally in the stomach. e.g. misroprostol, antacids etc.

- Drugs that are primarily absorbed in the stomach.

- Drugs which are poorly soluble in the alkaline Ph.

- Drugs that have narrow window of absorption in gastrointestinal tract e.g. L DOPA, para amino benzoic acids, furosemide, riboflavin etc.

- Drugs which are rapidly absorbed from the GI tract.

- Drugs that are degrade in the colon. Drugs those are unstable in the intestinal or colonic environment e.g. captopril, ranitidine HCL, metronidazole.

- Drugs that disturb normal colonic microbes e.g. antibiotics against Helicobacter pylori

In the design and development of hydro dynamically balanced system anatomical and physiological factors of stomach play an important role.

\section{CLASIFICATION OF GASTRO- RETENTIVE DOSAGE FORM $[10,14]$}

1) High density system

2) Floating system

3) Swelling system

4) Super porous hydro gels

5) Mucoadhesive system

6) Magnetic system

\section{High density system}

This approach involves formulation of dosage forms with density that must exceed density of normal stomach content $(1.004 \mathrm{~g} / \mathrm{ml})$. These types of formulations are prepared by coating drug on a heavy core or mixed with heavy inert material such as iron powder, zinc oxide, titanium dioxide, barium sulphate. The resultant pellets can be coated with diffusion controlled Membrane [35].

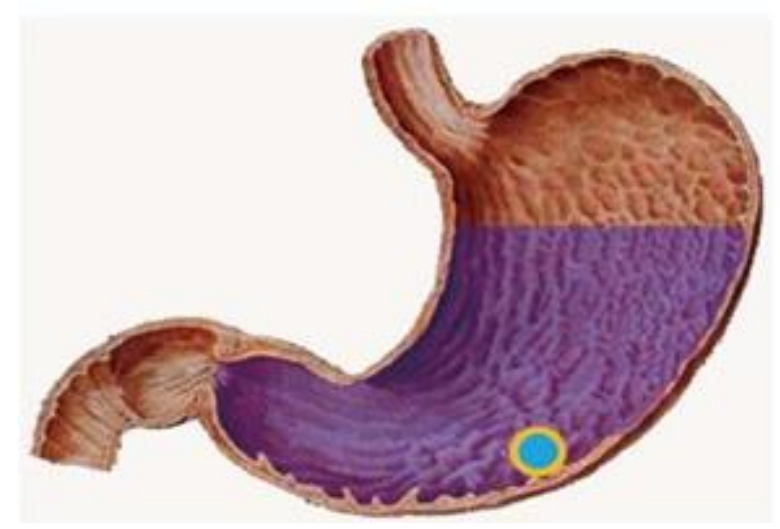

Figure 2. High density system

\section{Floating/low density system}

These types of dosage forms having density lower than that of gastric content, so FDDS remain float above the gastric contents for prolonged periods of time and provide continuous release of the drug. These systems in particular have been extensively studied because they do not adversely affect the motility of the GIT. Their dominance over the other types of GRRDS is also evident from the large number of floating dosage forms being commercialized and marketed worldwide.

\section{Classification of floating drug delivery system}

Floating system is classified as follows.

A) Effervescent system.

i) Gas generating system

- Single layer floating tablet

- Bi-layer floating tablet

- Multiple unit type pills 


\section{Indo Global Journal of Pharmaceutical Sciences, 2019; 9(1): 13-24}

- Floating system with ion exchange resin

ii) Volatile liquid containing system

- Intragastric gastrointestinal

- Inflatable gastrointestinal floating system

B) Non-effervescent system

i) Single layer floating tablet

ii) Bi-layer floating tablet

iii) Alginate beads

iv) Hollow microspheres

\section{A) Effervescent system}

Effervescent systems include use of gas generating agents, carbonates (e.g. Sodium bicarbonate) and other organic acid (e.g. citric acid and tartaric acid) present in the formulation to produce carbon dioxide $(\mathrm{CO} 2)$ gas, thus reducing the density of system and making it float on the gastric fluid. An alternative is the incorporation of matrix containing portion of liquid, which produce gas that evaporate at body temperature. These effervescent systems are divided into two parts i) gas generating system and ii) volatile liquid system

\section{i) Gas generating system}

These buoyant systems utilized matrices which are prepared with swell able polymers like HPMC, polysaccharides like chitosan, effervescent components like sodium bicarbonate, citric acid and tartaric acid or chambers containing a liquid that gasifies at body temperature. The optimal stoichiometric ratio of citric acid and sodium bicarbonate for gas generation is reported to be $0.76: 1$. The common approach for preparing these systems involves resin beads loaded with bicarbonate and coated with ethyl cellulose. The coating, which is insoluble but permeable, allows permeation of water. Thus, carbon dioxide is released, causing the beads to float in the stomach. Excipients used most commonly in these systems include HPMC, polyacrylate polymers, polyvinyl acetate, $\mathrm{Carbopol}{ }^{\circledR}$, agar, sodium alginate, calcium chloride, polyethylene oxide and polycarbonates.

These buoyant delivery systems utilize effervescent reactions between Carbonate/bicarbonate salts and citric/tartaric acid to liberate $\mathrm{CO} 2$, which gets entrapped in the jellified hydrocolloid layer of the systems thus decreasing its specific gravity and making it to float over gastric content [35,37].

\section{Hydro dynamically balanced system (HBS) or Single Layer Floating Tablet}

These are formulated by intimately mixing the $\mathrm{CO}_{2}$ generating agents and the drug within the matrix tablet. These have a bulk density lower than gastric fluids and therefore remain floating in the stomach unflattering the gastric emptying rate for a prolonged period. The drug is slowly released at a desired rate from the floating system and after the complete release the residual system is expelled from the stomach. This leads to an increase in the g.r.t. (gastric retention time) and a better control over fluctuation in plasma drug concentration.

\section{Bilayer Floating Tablets}

These are also compressed tablet as shown in Fig and containing two layer i.e. (1) Immediate release layer (2) Sustained release layer

\section{Multiple Unit Types Floating}

This system consists of sustained release Pills seeds surrounded by double layers. The inner layer consists of effervescent agents while the outer layer is of swell able membrane layer. When the system is immersed in dissolution medium at body temperature, it sinks at once and then forms swollen pills like balloons, which float as they have lower density. This lower density is due to generation and entrapment of $\mathrm{CO}_{2}$ within the systems.

\section{Floating system with Ion Exchange Resin}

Ion exchange resin, a multiple unit type of oral floating dosage system has been prepared to prolong the gastric emptying time of dosage form. The system is composed of beads of drugs resin complex, which are loaded with bi-carbonate ions and coated with a hydrophobic polymer, the system is so designed that when the beads reaches to the stomach chloride ions are exchanged with bicarbonate and drugs ions, the generated $\mathrm{CO}_{2}$ is entrapped in the polymeric coated resins and causes the beads to float.

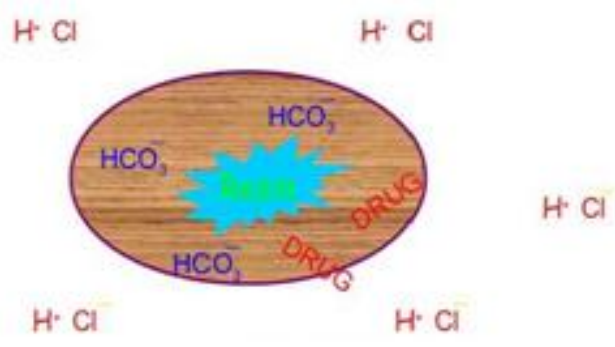

Figure 3. Ion exchange resin

\section{ii) Volatile liquid containing system}

The GRT of a drug delivery system can be sustained by incorporating an inflatable chamber which contains a liquid e.g. ether, cyclopentane, that gasifies at body temperature to cause the inflatation of the chamber in the stomach. The device may also consist of a bio erodible plug made up of Poly vinyl alcohol, Polyethylene etc. that gradually dissolves 


\section{Indo Global Journal of Pharmaceutical Sciences, 2019; 9(1): 13-24}

causing the inflatable chamber to release gas and collapse after a predetermined time to permit the spontaneous ejection of the inflatable systems from the stomach [36].

They are also divided into two parts.

\section{Intragastric floating gastrointestinal drug delivery system}

These systems can be made to float in the stomach because of floatation chamber, which may be a vacuum or filled with air or a harmless gas, while drug reservoir is encapsulated inside a micro porous compartment.

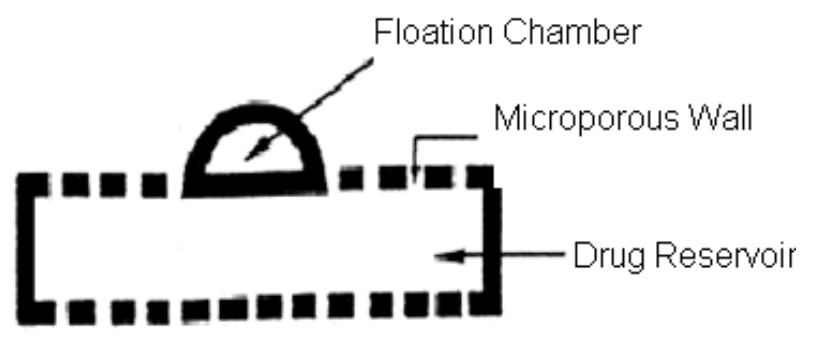

Figure 4. Intragastric Gloating Drug Delivery System

\section{Inflatable Gastrointestinal Delivery System}

In these systems an inflatable chamber is incorporated, which contains liquid ether that gasifies at body temperature to cause the chamber to inflate in the stomach. These systems are fabricated by loading the inflatable chamber with a drug reservoir, which can be a drug, impregnated polymeric matrix then encapsulated in a gelatin capsule. After oral administration, the capsule dissolves to release the drug reservoir together with the inflatable chamber. The inflatable chamber automatically inflates and retains the drug reservoir into the gastric fluid.

\section{Non-effervescent FDDS}

The Non-effervescent FDDS is based on mechanism of swelling of polymer or bio adhesion to mucosal layer in GI tract. The most commonly used excipients in non-effervescent FDDS are gel forming or highly swell able cellulose type hydrocolloids, hydrophilic gums, polysaccharides and matrix forming materials such as polycarbonate, polyacrylate, polymethacrylate, polystyrene as well as bioadhesive polymers such as Chitosan. The various type of this systems are as follows:

\section{i) Single layer floating system}

They are formulated by intimate mixing of drug with gelforming hydrocolloid, which
Swells in contact with gastric fluid and maintain bulk density of less than unity. The air trapped by the swollen polymer confers buoyancy to these dosage forms.

\section{ii) Bilayer floating tablet}

A bilayer tablet contain two layer immediate release layer which release initial dose from system while the another sustained release layer absorbs gastric fluid, forming an impermeable colloidal gel barrier on its surface, and maintain a bulk density of less than unity and thereby it remains buoyant in the stomach.

\section{iii) Alginate beads}

Multi-unit floating dosage forms have been developed from freeze dried calcium alginate. Spherical beads of approximately $2.5 \mathrm{~mm}$ in diameter can be prepared by dropping sodium alginate solution into aqueous solution of calcium chloride, causing the precipitation of calcium alginate. The beads are then separated, snap-frozen in liquid nitrogen, and freeze- dried at $-40^{\circ} \mathrm{C}$ for 24 hours, leading to the formation of a porous system, which can maintain a floating force for over 12 hours. These floating beads gave a prolonged residence time of more than 5.5 hours.

\section{iv) Hollow microspheres (Microbaloons)}

Hollow microspheres are considered as one of the most promising buoyant systems, as they possess the unique advantages of multiple unit systems as well as better floating properties, because of central hollow space inside the microsphere. Polymers such as polycarbonate, Eudragit ${ }^{\circledR} \mathrm{S}$ and cellulose acetate were used in the preparation of hollow microspheres. e. g. The research group of kawashima prepared hollow microspheres based on blends of Eudragit $S$ and HPMC

These microspheres were prepared by a novel emulsion solvent diffusion method. The ethanol/dichloromethane solution of the drug and an enteric acrylic polymer was poured into an agitated solution of Poly Vinyl Alcohol (PVA) that was thermally controlled at $40^{\circ} \mathrm{C}$. The gas phase is generated in the dispersed polymer droplet by the evaporation of dichloromethane formed and internal cavity in the microsphere of the polymer with drug. The micro balloon floated continuously over the surface of an acidic dissolution media containing surfactant for more than $12 \mathrm{~h}$.

\section{Swelling System}

These are the dosage forms, which after swallowing swells to such an extent that their exit from the pylorus prevented, as a result the dosage form is retained in the stomach for a prolonged period of time. These systems are called as plug - 


\section{Indo Global Journal of Pharmaceutical Sciences, 2019; 9(1): 13-24}

type system as they have the tendency to remain lodged at the pyloric sphincter .Controlled and sustained release may be achieved by selection of proper molecular weight polymer, and swelling of the polymers retard the release. On coming in contact with gastric fluid the polymer imbibes water and swells. The extensive swelling of these polymers is due to the presence of physical chemical cross links in the hydrophilic polymer network. These cross links prevents the dissolution of the polymer and hence maintain the physical integrity of the dosage form. In the dissolution media the membrane detached from the core and swelled to form a balloon that kept the unit floating. the size of the units increased by three to six folds, thus the floating ability as well as the increased dimension offered the system gastro retentive property

\section{Super porous hydro gels}

These are swell able systems that differ from conventional types. Absorption of water by conventional hydrogen is very slow process and several hours may be required to reach the equilibrium states [41] during which the premature evacuation of the dosage form may occur. Super porous hydro gel have a pore size $>100 \mu \mathrm{m}$ which swell to equilibrium size with in a minutes, due to rapid intake of water by capillary wetting through inter connected open pores. They swell to a larger size and have sufficient mechanical strength to withstand the pressure by gastric contraction. This is achieved by coformulation of a hydrophilic particulate material, Ac-Di- Sol.

\section{Mucoadhesive systems}

Mucoadhesive drug delivery systems contain a mucoadhesive polymer that adheres to the gastric mucosal surface and prolong its gastric retention in the g.i.t. The capability to adhere to the mucus gel layer makes mucoadhesive polymers very useful exicipients in the GRRDS. These polymers can be natural such as sodium alginate, gelatin, guar gum etc semi synthetic polymers such as HPMC, carbopol, sodium carboxymethyl cellulose the adhesion of polymers with mucous membrane may be mediated by hydration bonding, or receptor mediated. In hydration mediated adhesion, the hydrophilic polymer become sticky and mucoadhesive upon hydration. Bonding mediated involves mechanical or chemical bonding. Chemical bonds may involve ionic or covalent bonds or Vander Waal forces between the polymer molecule and the mucous membrane. Receptor mediated adhesion takes place between certain polymers and specific receptors expressed on gastric cells. The polymers can be cationic or anionic or neutral. a) Hydration - mediated adhesion

Certain hydrophilic polymers have the tendency to imbibe large amount of water and become sticky, thereby acquiring bioadhesive properties. The prolonged gastro retention of the bio/muco-adhesive delivery system is further controlled by the dissolution rate of the polymer.

\section{b) Bonding mediated adhesion}

Adhesion of polymers to mucus/epithelial cell surface involves varying bonding mechanism. Physical or mechanical bonds can result from deposition and inclusion of the adhesive material in the crevices of the mucosa. Secondary chemical bonds, contributing to bioadhesive properties, consist of dispersive interactions (i.e. van der Walls interactions) and stronger specific interaction, which include on the cell surface. The receptor mediated hydrogen bonds. The hydrophilic functional groups responsible for forming hydrogen bonds are the hydroxyl (--OH) and the carboxylic groups (--COOH) [37]

\section{c) Receptor mediated adhesion}

Certain polymers have the ability to bind to specific receptor sites events serves as a potential approach in bio/mucoadhesion, hence enhancing the gastric retention of dosage forms. Certain plant lectins, like tomato lectins interact specifically with the sugar groups present in mucus or on the glycocalyx.

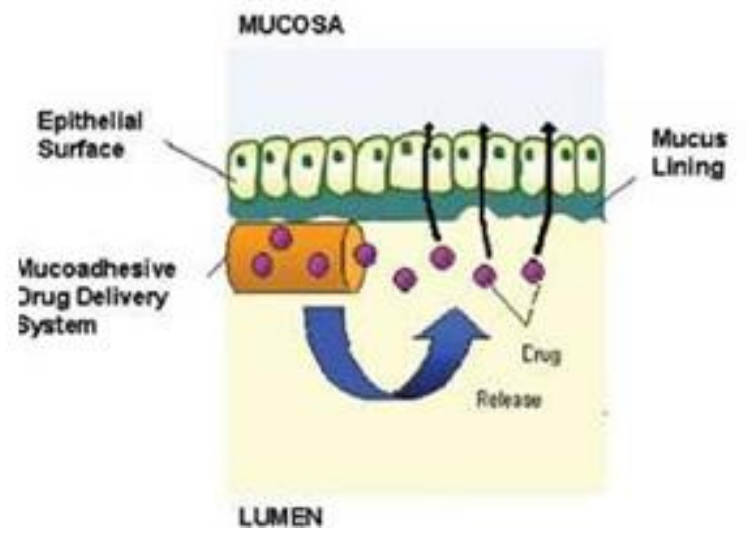

Figure 5. Mucoadhesive system.

\section{Magnetic system}

In an increased GRT and a better control of the fluctuations in plasma drug concentration This system is based on the simple idea that the dosage form contains a small internal magnet, and a magnet placed on the abdomen over the position of the stomach. Using an extracorporeal magnet, gastric residence time of the dosage form can be enhanced for a prolonged period of time. 


\section{Indo Global Journal of Pharmaceutical Sciences, 2019; 9(1): 13-24}

\section{MECHANISM OF FLOATING SYSTEM [15]}

Floating drug delivery systems (FDDS) have a bulk density less than gastric fluids and so remain buoyant in the stomach without affecting the gastric emptying rate for a prolonged period of time. While the system is floating on the gastric contents (Fig.: 1) the drug is released slowly at the desired rate from the system. After release of drug, the residual system is emptied from. However, besides a minimal gastric content needed to allow the proper achievement of the buoyancy retention principle, a minimal level of floating force $(\mathrm{F})$ is also required to keep the dosage form reliably buoyant on the surface of the meal. To measure the floating force kinetics, a novel apparatus for determination of resultant weight has been reported in the literature. The apparatus operates by measuring continuously the force equivalent to $\mathrm{F}$ (as a function of time) that is required to maintain the submerged object.

The object floats better if $\mathrm{F}$ is on the higher positive side. This apparatus helps in optimizing FDDS with respect to stability and durability of floating forces produced in order to prevent the drawbacks of unforeseeable intra-gastric buoyancy capability variations ${ }^{17}$.

$$
\mathrm{F}=\mathrm{F}_{\text {buoyancy }}-\mathrm{F}_{\text {gravity }}=(\mathrm{Df}-\mathrm{Ds}) \mathrm{gV}
$$

Where, $\mathrm{F}=$ total vertical force, $\mathrm{Df}=$ fluid density, $\mathrm{Ds}=$ object density, $\mathrm{V}=$ volume and $\mathrm{g}=$ acceleration due to gravity
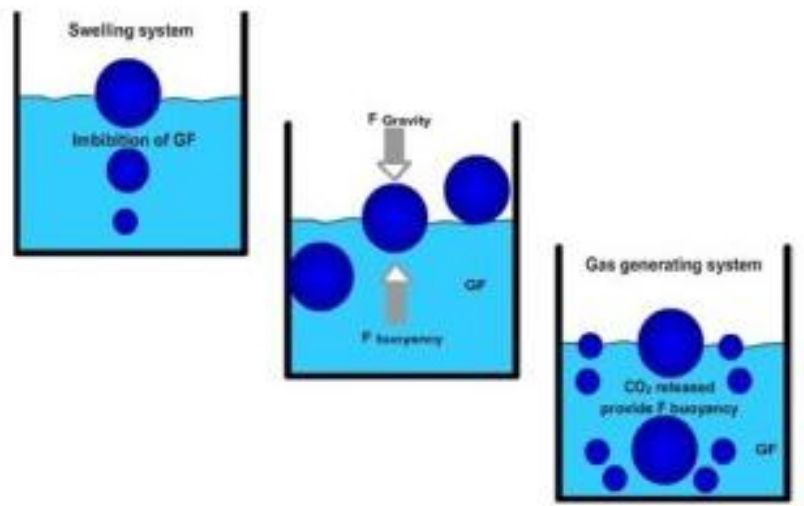

Figure 6. Mechanism of floating drug delivery system

\section{DEVELOPMENTAL APPROACHES OF FLOATING MICROSPHERES [16,}

\section{$17,18]$}

Wide ranges of developmental techniques are available for the preparation of gastro retentive floating microspheres.
However, solvent evaporation technique and ion tropic gelation method have been extensively employed by large number of scientific investigators worldwide to explore the different vistas of floating microspheres. During the preparation of floating controlled release microspheres, the choice of optimal method has utmost relevance for the efficient entrapment of active constituents. Selection of fabrication technique generally depends upon the nature of the polymer, the drug, and their intended use . Characteristic features of materials and the process engineering aspects strongly influence the properties of microspheres and the resultant controlled release rate. These techniques (i.e. solvent evaporation and ion tropic gelation)

\section{a) Solvent Evaporation Technique [19,20]}

This technique is widely employed by large number of pharmaceutical industries to obtain the controlled release of drug. This approach involves the emulsification of an organic solvent (usually methylene chloride) containing dissolved polymer and dissolved/dispersed drug in an excess amount of aqueous continuous phase, with the aid of an agitator. The concentration of the emulsifier present in the aqueous phase affects the particle size and shape. When the desired emulsion droplet size is formed, the stirring rate is reduced and evaporation of the organic solvent is carried out under atmospheric or reduced pressure at an appropriate temperature. Subsequent evaporation of the dispersed phase solvent yields solid polymeric micro particles entrapping the drug. The solid micro particles are recovered from the suspension by filtration, centrifugation, or lyophilisation. For emulsion solvent evaporation, there are basically two systems which include oil-in-water (o/w) and water-in-oil (w/o) type.

\section{b) Oil-In-Water Emulsion Solvent Evaporation Technique [21-24]}

In this process, both the drug and the polymer should be insoluble in water while a water immiscible solvent is required for the polymer. In this method, the polymer is dissolved in an organic solvent such as dichloromethane, chloroform, or ethyl acetate, either alone or in combination. The drug is either dissolved or dispersed into polymer solution and this solution containing the drug is emulsified into an aqueous phase to make an oil-in water emulsion by using a surfactant or an emulsifying agent. After the formation of a stable emulsion, the organic solvent is evaporated either by increasing the temperature under pressure or by continuous stirring. Solvent removal from embryonic microspheres determines the size and morphology of the microspheres. It has been reported that the rapid removal of solvent from the embryonic microspheres leads to polymer precipitation at the $\mathrm{o} / \mathrm{w}$ interface. This leads to the formation of cavity in microspheres, thus making them 


\section{Indo Global Journal of Pharmaceutical Sciences, 2019; 9(1): 13-24}

hollow to impart the floating properties .Oil-in water emulsion is widely used than water-in-oil due to simplicity of the process and easy cleans up requirement for the final product

\section{c) Oil-in-Oil Emulsification Solvent Evaporation Technique $[25,26]$}

This oil-in-oil (sometimes referred as water-in-oil) emulsification process is also known as non aqueous emulsification solvent evaporation. In this technique, drug and polymers are dissolve at room temperature into polar solvents such as ethanol, dichloromethane, acetonitrile etc. with vigorous agitation to form uniform drug-polymer dispersion. This solution is slowly poured into the dispersion medium consisting of light/heavy liquid paraffin in the presence of oil soluble surfactant such as Span. The system is stirred using an overhead propeller agitator at 500 revolutions per minute (rpm) and room temperature over a period of $2-3 \mathrm{~h}$ to ensure complete evaporation of the solvent. The liquid paraffin is decanted and the micro particles are separated by filtration through a Whatmann filter paper, washed thrice with nhexane, air dried for $24 \mathrm{~h}$ and subsequently stored in desiccators. Span 60 is generally used which is non ionic surfactant. Span 60 has an HLB value of 4.3 and acts as a droplet stabilizer and prevents coalescence of the droplets by localizing at the interface between the dispersed phase and dispersion medium.

\section{d) Ionotropic Gelation Method[27,28]}

In this method, cross linking of the polyelectrolyte takes place in the presence of counter ions to form gel matrix. This technique has been generally employed for the encapsulation of large number of drugs. Polyelectrolyte such as sodium alginate having a property of coating on the drug core and acts as release rate retardant contains certain anions in their chemical structure. These anions forms meshwork structure by combining with polyvalent cations and induced gelation. Microspheres are prepared by dropping drug loaded polymeric solution using syringe into the aqueous solution of polyvalent cations as depicted in. The cations diffuses into the drug loaded polymeric drops, forming a three dimensional lattice of ionically cross linked moiety. Microspheres formed are left into the original solution for sufficient time period for internal gelification and they are separated by filtration. Natural polymers such as alginates can be used to improve drug entrapment and are widely used in the development of floating microspheres.

\section{POLYMERS USED IN FLOATING DRUG DELIVERY SYSTEM [37]}

Polymers play a very important role in Controlled drug delivery system. And FDDS is an approach to achieve drug release for long period of time. So, Polymers, which can be successfully used in floating drug delivery system, are briefly discussed here.

Acrylic polymers are widely used for the preparation of floating microspheres, because of good floating behavior, slow dissolution rate, low solubility of polymers in acidic $\mathrm{pH}$. I.e. Eudragit E100 and Eudragit RL 30D.

For floating purpose the dosage forms must have the following properties

- It should release contents slowly to serve as a reservoir.

- It must maintain specific gravity lower than gastric contents $(1.004-1.01 \mathrm{gm} / \mathrm{cm} 3)$.

- It must form a cohesive gel barrier. The inherent low density can be provided by the entrapment of air (e.g. hollow chambers) or by the incorporation of low density materials (e.g. fatty materials or oils, or foam powder). These following approaches have been used for the design of floating dosage forms of single and multiple-unit dosage forms. Recently a single unit floating system was proposed consisting of polypropylene foam powder, matrix forming polymers, drug and filler. The good floating behavior of these systems could be successfully combined with accurate control of the resulting drug release patterns. Single-unit dosage forms are associated with problems such as sticking together or being obstructed in the gastrointestinal tract (GIT) which may produce irritation. On the other hand multiple-unit floating systems may be an attractive alternative since they have been shown to reduce the inter- and intra- subject availabilities in drug absorption as well as to lower the possibility of dose dumping. Various multiple-unit floating system like air compartment multiple-unit system, hollow microspheres (microballoons) prepared by the emulsion solvent diffusion method, microparticles based on low density foam powder, beads prepared by emulsion gelatin method etc. can be distributed widely throughout the GIT, providing the possibility of achieving a longer lasting and more reliable release of drugs. Based on the mechanism of buoyancy two distinctly different technologies, i.e. non-effervescent and effervescent systems have been utilized in the development of floating drug delivery system.

\section{CHARACTERIZATION OF PREPARED MICROSPHERES}

The microspheres were characterized for their particle size, bulk and tapped densities, compressibility index and angle of repose as described below. 


\section{Indo Global Journal of Pharmaceutical Sciences, 2019; 9(1): 13-24}

1) Micromeritics properties $[29,30]$

Floating microspheres are characterized by their micromeritics properties such as particle size, Flow property and Density. Angle of Repose, Hausner's Ratio, and compressibility index is determined by measuring the change in volume using a bulk density apparatus; angle of repose is determined by fixed funnel method. The hollow nature of microspheres is confirmed by scanning electron microscopy.

\section{a) Particle size}

The particle size of the microspheres was measured using an optical microscopic method and mean microsphere size was calculated by measuring 100 particles with the help of a calibrated ocular micro meter.

\section{b) Bulk density}

Bulk density is defined as the mass of powder divided by bulk volume. Accurately weighed $10 \mathrm{gm}$. sample of granules was placed into $25 \mathrm{ml}$ measuring cylinder. Volume occupied by the granules was noted without disturbing the cylinder and the bulk density was calculated using the equation (values expressed in $\mathrm{gm} / \mathrm{cm} 3$ )

Dry bulk density $=$ mass of soil/ volume as a whole

\section{c) Tapped density}

The tapping method can be used to calculate tapped densities. The volume of weighed quantity of microspheres was determined after 100 taps as well as 1000 taps using tapped density apparatus

Tapped density = Mass of microspheres/ Volume of microspheres after tapping

\section{e) Compressibility Index}

It can be calculated from the values of bulk density and tapped density by using following formulas.

$$
\mathrm{I}=\mathrm{V}_{\mathrm{b}}-\mathrm{V}_{\mathrm{t}} / \mathrm{V}_{\mathrm{b}} \times 100
$$

Where $\mathrm{V}_{\mathrm{b}}$ is the bulk volume and $\mathrm{Vt}$ is the tapped volume, the value given below the $15 \%$ indicates a powder with usually give rise to good flow characteristics, whereas above $25 \%$ indicates poor flow ability.

\section{f) Angle of Repose}

The angle of repose $\theta$ of the microspheres, which measures the resistance to particle flow, was calculated as

$\operatorname{Tan} \theta=\mathrm{h} / \mathrm{r}$ Therefore,

$$
\theta=\tan -1 \mathrm{~h} / \mathrm{r}
$$

Where, $\theta$ is angle of repose, $h$ is height of the pile; $r$ is the radius of the pile.

\section{2) Percentage yield}

Percentage yield of floating microspheres was calculated by dividing actual weight of product to total amount of all non-volatile components that are used in the preparation of floating microspheres and is represented by following formula. $\%$ yield $=$ (actual weight of product/total weight of drug and Excipients) $\times 100$ 7. Drug entrapment efficiency (DEE)15 The amount of drug entrapped was estimated by crushing the microspheres and extracting with aliquots of $0.1 \mathrm{~N} \mathrm{HCl}$ repeatedly. The extract was transferred to a $100 \mathrm{ml}$ volumetric flask and the volume was made up using $0.1 \mathrm{~N} \mathrm{HCl}$. The solution was filtered and the absorbance is measured by spectrophotometer against appropriate blank. The amount of drug entrapped in the microspheres was calculated by the following formula: $\mathrm{DEE}=$ (amount of drug actually present/theoretical drug load expected) $\times 100$

\section{3) Floating behavior [31]}

Appropriate quantity of the floating microspheres were placed in $100 \mathrm{ml}$ of the simulated gastric fluid (SGF, pH 2.0), the mixture was stirred with a magnetic stirrer. The layer of buoyant micro particulate was pipette and separated by filtration. Particles in the sinking particulate layer were separated by filtration. Particles of both types were dried in a desiccators until constant weight was achieved. Both the fractions of microspheres were weighed and buoyancy was determined by the weight ratio of floating particles to the sum of floating and sinking particles.

$$
\text { Buoyancy }(\%)=\mathrm{W}_{\mathrm{f}} / \mathrm{W}_{\mathrm{f}}+\mathrm{Ws}_{\mathrm{s}}
$$

Where, $\mathrm{W}_{\mathrm{f}}$ and $\mathrm{Ws}$ are the weights of the floating and settled micro particles

\section{4) \%Drug entrapment [32]}

Accurately weighed microspheres were taken, thoroughly triturated and suspended in a minimal amount of solvent. The suspension was filtered to separate shell fragments. Drug contents were analyzed and \% Drug entrapment is calculated by using following equation.

$\%$ Drug Entrapment= Actual drug content $/$ Theoretical drug content $\times 100$ 


\section{Indo Global Journal of Pharmaceutical Sciences, 2019; 9(1): 13-24}

\section{5) In-vitro release studies [32]}

The release rate of floating micro particulate was determined in dissolution apparatus. A weighed amount of floating microspheres equivalent to Dose of drug is taken and placed in the basket type of dissolution test apparatus. The dissolution fluid was maintained at $37 \pm 1{ }^{\circ} \mathrm{C}$ at a rotation speed. Perfect sink conditions prevailed during the drug release study.

\section{6) In-vivo studies [33]}

The in-vivo floating behavior can be investigated by X-ray photography of hollow micro particulate loaded with Barium sulphate in the stomach of beagle dogs. The in vitro drug release studies are performed in a dissolution test in a dissolution media. The in-vivo plasma profile can be obtained by performing the study in suitable animal models.

\section{LIMITATIONS OF FLOATING DRUG DELIVERY SYSTEM [34]}

1) The major limitation of floating system is the requirement of sufficient high level fluids in the stomach for the drug delivery to float. But this limitation can be overcome by coating the tablet with bioadhesive polymers that easily adhere to the mucosal lining of the stomach.

2) The retention time in the stomach depends upon the digestive state. Hence FDDS should be administered after the meal.

3) The ability to float depends on the hydration state of the dosage form, In order to keep these tablets floating in-vivo, intermittent administration of water (A tumbler full, every 2 hours) is beneficial.

4) The retention of the dosage form in the stomach depends upon the subject being position upright.

5) FDDS are not suitable for the drugs that have solubility and stability problems in the gastric fluids.

6) Drugs like nifedipine, which under goes significant fist pass metabolism is not a desirable candidate for FDDS, since the slow gastric emptying may lead to the reduced systemic bioavailability.

\section{CONCLUSION}

Drug absorption in the gastrointestinal tract is a highly variable procedure and prolonging gastric retention of the dosage form extends the time for drug absorption.
Hollow microsphere promises to be a potential approach for gastric retention. Although there are number of difficulties to be worked out to achieve prolonged gastric retention, a large number of companies are focusing toward commercializing this technique.

\section{REFERENCES}

1. Microspheres, Novel drug delivery systems, Vol. 50, Marcel Dekker, Inc., New York, 1992; 50: 139-177.

2. Patel, Geetha. M, Floating Drug Delivery System: An Innovative Approach to Prolong Gastric Retention, www.Pharmainfo.net, 2007; Vol:5(6).

3. Rahaman Z., Ali M, Khar RK. Design and evaluation of bilayer floating tablets of captopril. Acta Pharma. 2006; Vol:56(1): pg- 49-57.

4. Tanwar YS Naruka PS, Ojha GR., Development and evaluation of floating microspheres of verapamil hydrochloride. Brazilian J Pharm Sci. 2007; Vol:43(4): pg- 529-534.

5. Lee TW, Robinson JR, Remington: The Science and Practice of Pharmacy.20th Ed.Pennsylvania. Mack Publishing Company 2001.

6. Shwetha Kamath K. Senthil Kumar S.K. Design and evaluation of floating microspheres of Rabeprazole Sodium. International Journal of Pharmacy and Pharmaceutical sciences, 2012; Vol: 4(3); Pg-357-367.

7. Gadad A., Naval C., Patel K., Dandagi P. and Vinaya. Formulation and evaluation of floating microspheres of captopril for prolonged gastric residence time. Indian Journal of Novel Drug Delivery, 2011; Vol:3(1), pg-17-23.

8. Deshpande, A. A.,Shah, N.H., Rhodes, C.T., Malick, W., Development of a novel controlled release system for gastric retention, Pharm. Res. 1997; 14 (6): 815-9.

9. Atyabi, F., Sharma, H.L., Mohammad, H., Fell, J. T., In-vivo evaluation of a novel gastroretentive formulation based on ion exchange resins, $\mathrm{J}$. Control. Rel. 1996; 42: 105-113.

10. Nayak A.k., Mazi.R.,Das B., Gastroretentive drug delivery system a review, Asian journal of pharmaceutical and clinical research,Vol 3,issue 1, January-march 2010, ISSN 0974-2441

11. Ojha G., Tanwar Y.S., CHuhan C.S., Naruka P.S., Floating microspheres, development, characterization and applications. http;///www.pharmainfo.net/volume-and issues/2006/vol-4-issue3.

12. Badoni A., Ojha A., Gnanarajani G., Kothiyali P.; Review on Gastro Retentive Drug Delivery System; The Pharma Innovation, 2012;1(8): 32-42.

13. DR. Jose G.R., Omidien H. Shah K.Progress in gastroretentive drug delivery system. Pharmtech 152-160.(2003)

14. Jassal M., Nautiyal U., Kundlas J., Singh D., a Review on gastro retentive drug delivery system, Indian J. Pharm. Biol. Res.2015; 3(1):82-92, ISSN: 2320-9267

15. Mayavanshi A.V. and Gajjar SS: Floating drug delivery system to increase gastric retention of drugs: A review. Research Journal of Pharmaceutical Technology 2008; 1(4): 345-48.

16. Benita S. In: Microencapsulation, New York: Marcel Dekkar; 1996; p 121.

17. Okada H, Toguchi H. „Critical Reviews in Therapeutics Drug Carrier Systems ${ }^{\text {ee }}$. 1995; . 12(1): 1-99.

18. Khar R.K, Vyas S.P. „Targeted and controlled drug delivery novel carrier

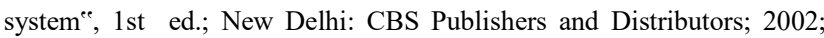
417- 441.

19. Li M, Rouaud O, Poncelet D. Microencapsulation by solvent evaporation: state of the art for process engineering approaches Inter J Pharm. 2008; 363(1-2): 26-39

20. Watts PJ, Davis MC, Melia CD. „Microencapsulation using emulsification solvent evaporation: an overview of techniques and applications ${ }^{\text {ee }}$. Crit Rev Ther Drug Carrier. 
Indo Global Journal of Pharmaceutical Sciences, 2019; 9(1): 13-24

21. Jalil R, Nixon JR, Biodegradable poly (lactic acid) and poly (lactide-coglycolide) microcapsules: problems associated with preparative techniques and release properties Journal of Microencapsulation. 1990; 7(3): 297-325.

22. Lee JH, Park TG, Choi HK. Development of oral drug delivery system using floating microspheres. J Microencapsul. 1999; 16(6): 715-729.

23. Garg R, Gupta G.D., Gastroretentive Floating Microspheres of Silymarin: Preparation and In Vitro Evaluation Trop J Pharm Res. 2010; 9(1): 59-66.

24. Huang H.P, Ghebre-sellassie I., Preparation of microspheres of watersoluble pharmaceuticals. J Microencapsul. 1989; 6(2): 219-225

25. Gattani Y.S, Bhagwat D.A, Maske A.P., „Formulation and evaluation of intragastric floating drug delivery system of diltiazem hydrochloride"e. Asian J Pharm. 2008; 2(4): 228-231.

26. Hincal A.A, Calis S. „In: Handbook of Pharmaceutical Controlled Release Technology". New York: Marcel Dekker. 2005; 329 -343

27. Sam T.M., Gayathri DS, Prasanth V.V., Vinod B. The Inter J Pharmaco. 2008; 6(1):21- 32

28. Patil J.S., Kamalapur M.V., Marapur S.C., Kadam D.V. Dig J Nano and Bio. 2010; 5(1): 241-248.

29. Martin. A, Swarbrick J, Cammarata A. Physical Pharmacy, III Ed, Varghese Publishing Company, Bombay. 1991; 492- 520.

30. Umamaheshwari RB, Jain S, Bhadra D, Jain NK. Floating for the treatment of Helicobacter pylori. Int. J. Pharm. 2003; 55(12): 1607-1613.

31. Jain NK. Progress in Controlled and Novel Drug Delivery Systems, 1Ed. CBS Publishers and Distributors, New Delhi, Bangalore, 2004; 84-85.

32. Gholap SB, Banarjee SK, Gaikwad DD, Jadhav SL, Thorat RM. Hollow microsphere. A review. Int J Pharmacy and Pharm Sci. March-April 2010. 1(1), 210-220.

33. Whitehead L, Fell JT, Collett JH, Sharma HL, Smith A. In-vivo study demonstrating prolonged gastric retention. J. Con. Rel. 1998; 55: 312.

34. Guttani Y.S., Floating multiparticulate drug delivery system; an overview, ijpbs Vol 1, (2010)

35. Shan Z., Yan L., Jian B.Z., Bing W., Guo-J.L., Xiao J. M. Gastro retentive drug delivery system for the treatment of Helicobacter pylori, world J gastroenterol, july 2014;20(80), 9321-9329.

36. Kamini, Kaur M., Pooni N., Singh M., Verma K., Dhiman N., Sharma N., Bhandari N., Floating drug delivery system a review, World Journal of Pharmacy and Pharmaceutical Sciences, Volume 6, Issue 4, 578594,ISSN $2278-4357$.

37. Solanki D., Patidar S., Motiwale M., Kushwah L., Floating drug delivery system -novel tool for delivering $\mathrm{H} 2$ antagonist. International journal of pharmacy and pharmaceutical research 2017, ISSN2349-7203.

38. Pawar V.K., Shaswat K., Garg G., Awasthi R., Singodia D. \& KulkarniG.T. Gastroretentive dosage forms: A review with special emphasis on floating drug delivery systems, Drug Delivery, 2011; 18(2): 97-110. ID: 101610675) indexed and abstracted in CrossRef (DOI Enabling), UGC CARE Journal List, EMBASE(Elsevier), National Library of Medicine (NLM) Catalog, ResearchGate, Publons, CAS (ACS), Index Copernicus, Google Scholar and many more. For further details, visit http://iglobaljournal.com 